\title{
Identification of Bacillus spp. colonizing the nasal mucosa of healthy adults living in the suburban area using the matrix-assisted laser desorption-ionization time-of-flight mass spectrometry (MALDI-TOF MS) system
}

\author{
Urszula Kosikowska ${ }^{1 \star}$, Dagmara Stepien-Pysniak ${ }^{2}$, Dorota Ozga ${ }^{3}$, \\ ANDRZEJ WernicKI ${ }^{2}$, ANNA MALM ${ }^{1}$
}

\begin{abstract}
${ }^{1}$ Department of Pharmaceutical Microbiology, Medical University of Lublin, W. Chodzki 1, 20-093 Lublin, Poland
${ }^{2}$ Sub-Department of Veterinary Prevention and Avian Diseases, Institute of Biological Bases of Animal Diseases, Faculty of Veterinary Medicine, University of Life Sciences of Lublin, Gleboka 30, 20-612 Lublin, Poland

${ }^{3}$ Department of Epizootiology and Clinic of Infectious Diseases, Faculty of Veterinary Medicine, University of Life Sciences of Lublin, Gleboka 30, 20-612 Lublin, Poland
\end{abstract}

\begin{tabular}{l}
\hline ARTICLE INFO \\
\hline Received 1 October 2014 \\
Accepted 20 October 2014 \\
\hline
\end{tabular}

Keywords:

Bacillus spp.,

nasal mucosa,

MALDI-TOF MS

\begin{abstract}
Bacillus spp. can be regarded as a rare component of the nasal mucosa microflora.

The aim of this study was to identify Bacillus spp. from the nasal mucosa of healthy adults living in the suburban area near Lublin using the matrix-assisted laser desorptionionization time-of-flight mass spectrometry (MALDI-TOF MS) system.

A total of 11 bacterial isolates from the nasal specimens were cultured. The following species were identified using the routine microbiological methods: Staphylococcus aureus (3 isolates), S. epidermidis (1 isolate), S. intermedius (1 isolate) and Staphylococcus spp. (1 isolate). Moreover, 2 strains of Escherichia coli were isolated. Besides, 3 isolates of Bacillus spp. were found. These isolates were characterized by means of MALDI-TOF MS, resulting in highly specific mass spectral fingerprints and these were identified as B. pumilus, B. safenis and B. licheniformis. It was observed that all studied Bacillus spp. isolates only had the masses in common at $3864 \pm 2,7727 \pm 2$, and $14301 \pm 4$. The spectra of B. safensis and B. pumilus showed peaks at $\mathrm{m} / \mathrm{z} 4914 \pm 3,6621 \pm 3$ and $14291 \pm 2$, which were absent in the spectrum of B. licheniformis. For B. safenis and B. pumilus, other potential biomarkers could be found at $m / z 12620$ and 16668, respectively.
\end{abstract}

\section{INTRODUCTION}

The microbiota of the upper respiratory tract mucosa composition is rich and extremely diverse. A typical nasal microflora is composed of staphylococci, including S. aureus and coagulase-negative staphylococci, mainly $S$. epidermidis [13]. Besides, other bacteria like Bacillus spp. or Gramnegative rods, e.g. E. coli may be a part of this microflora. People living in suburban areas are often exposed to the presence of many opportunistic environmental pathogens, being then the colonizers of the human body. Routine methods of microbiological diagnostics together with commercial tests allow identifying only the most frequently isolated microorganisms $[8,15,20,22]$. Nowadays a growing

\section{Corresponding author}

e-mail: urszula.kosikowska@umlub.pl incidence of Bacillus spp. is observed in several clinical specimens where they are not the contamination $[9,25]$. This may cause diagnostic problems and require the use of more sophisticated methods not available in medical laboratories. Here we describe the matrix-assisted laser desorption-ionization time-of-flight mass spectrometry (MALDI-TOF MS) platform for the rapid discrimination of Bacillus spp. isolates and other selected bacteria isolated from the nasal mucosa of healthy adults living in the suburban area near Lublin.

\section{MATERIALS AND METHODS}

In the present study conducted between June and July 2014 , bacterial isolates from the nasal mucosa of 7 healthy adults living in the neighborhood in the suburban area near Lublin, Poland (the Ethics Committee Medical University 
of Lublin approved the study protocol, KE-0254/75/2011) were included. A total of 14 swabs were collected from the nasal mucosa of both nostrils of each person. The specimens were cultured on routinely used media (agar, blood agar, Chapman and McConkey media) at $35^{\circ} \mathrm{C}$ for $24-48 \mathrm{~h}$. The bacterial isolates were initially identified based on the morphology of their colonies, Gram stain morphology and/ or biochemical methods (API 20E and ID 32 Staph systems, bioMerieux, France). Eleven isolates were included in the analysis by MALDI-TOF MS system (Daltonik Bruker, Germany). The reference species of Escherichia coli ATCC 25922 and Staphylococcus epidermidis ATCC 12228 were chosen as positive controls for the MALDI-TOF MS evaluation because they are representatives of the microflora of the nasal mucosa.

All samples for mass spectrometry were prepared as recommended by the manufacturer $[15,35]$. The identification was preceded by preliminary extraction of proteins with ethanol and formic acid. For this purpose, a single colony of a fresh 18-24 hour culture growing on agar medium at $35^{\circ} \mathrm{C}$ was suspended in $300 \mu \mathrm{l}$ of sterile deionized water, after which $900 \mathrm{ml}$ of pure ethanol (POCH) was added. Then each sample was mixed thoroughly by vortexing. The resulting sample was then centrifuged for 2 minutes at 13000 $\mathrm{rpm} / \mathrm{min}$. After the supernatant had been discarded, $50 \mathrm{ml}$ of $70 \%$ aqueous formic acid and then $50 \mathrm{ml}$ of acetonitrile (Fluka Analytical, Switzerland) were added to the precipitate and the sample was thoroughly mixed by vortexing. After centrifugation (13 $000 \mathrm{rpm} / \mathrm{min}$ for $2 \mathrm{~min}), 1 \mu \mathrm{l}$ of the supernatant was collected, applied on a metal plate and allowed to dry at room temperature. Then $1 \mu$ of matrix solution was applied and the sample was left to dry at room temperature. The metal plate with the samples was placed in a MALDI Biotyper chamber for analysis. An automatic measurement of the spectrum and a comparative analysis with reference spectra of bacteria were performed using the Ultraflextreme mass spectrometer and MALDI-Biotyper 3.0 software (Bruker Daltonik, Germany), including 3672 spectra. The reliability of identification in the MALDIBiotyper 3.0 software was expressed in points. A score $\geq 1.7$ and $<2.0$ indicated identification to the genus level, while scores $\geq 2.0$ indicated identification to the species level. The similarity values required to separate Bacillus spp. species on the basis of the protein profile were according to MALDI Biotyper 3.0 software spectra.

\section{RESULTS}

A total of 11 bacterial species were isolated from the nasal mucosa membrane of healthy adults living in the suburban area near Lublin (Table 1). In the diagnostic workflow of the isolates, the majority of bacterial species were analysed and identified by routine diagnostic methods and all of them by MALDI-TOF MS system. Routine diagnostic methods revealed the occurrence of 6 isolates of staphylococci: S. aureus (3 isolates), S. epidermidis (1 isolate), S. intermedius (1 isolate), Staphylococcus spp. (1 isolate). In addition, 2 isolates of $E$. coli were found. Besides, 3 isolates of Bacillus spp. were identified using colony morphology and Gram-staining (Figure 1)

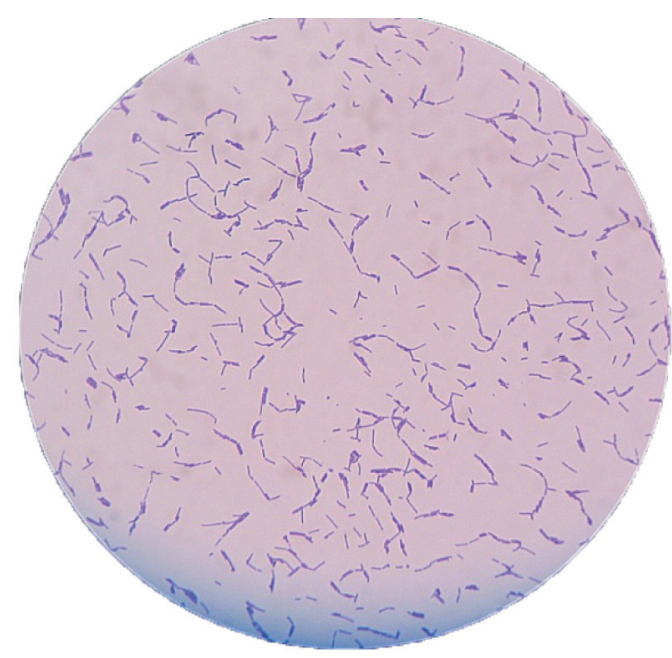

Figure 1. A microscopic image of Bacillus spp. isolate from the nasal mucosa of healthy adults staining by the Gram method

All Bacillus spp. isolates were analysed after formic acid extraction by MALDI-TOF MS (Table 1). Among these isolates $B$. licheniformis (1 isolate), B. pumilus (1 isolate) and B. safensis (1 isolate) were identified. Besides, 1 isolate of Staphylococcus spp. was identified as S. pseudintermedius.

Table 1. Prevalence of bacterial isolated from nasal mucosa of healthy adults living in the suburban area

\begin{tabular}{|c|c|c|c|}
\hline \multirow{2}{*}{ No. } & \multirow{2}{*}{ Sex } & \multicolumn{2}{|c|}{ Identification of isolates by using } \\
\cline { 3 - 4 } 1 & F & routine diagnostic methods & MALDI-TOF MS \\
\hline 2 & F & Bacillus spp. & Bacillus licheniformis \\
\hline 3 & F & Staphylococcus aureus & Staphylococcus aureus \\
\hline 4 & M & Staphylococcus aureus & Staphylococcus aureus \\
\hline \multirow{2}{*}{6} & \multirow{2}{*}{ M } & Staphylococcus intermedius & Staphylococcus intermedius \\
\cline { 3 - 4 } & & Staphylococcus spp. & Staphylococcus pseudintermedius \\
\hline \multirow{2}{*}{5} & \multirow{2}{*}{ M } & Bacillus spp. & Bacillus safensis \\
\cline { 3 - 4 } & & Staphylococcus epidermidis & Staphylococcus epidermidis \\
\hline \multirow{2}{*}{7} & \multirow{2}{*}{ M } & Staphylococcus aureus & Staphylococcus aureus \\
\cline { 3 - 4 } & & Escherichia coli & Escherichia coli \\
\cline { 3 - 4 } & & Escherichia coli & Escherichia coli \\
\hline
\end{tabular}

Note: $\mathrm{F}$ - female, $\mathrm{M}$ - male

The results of MALDI-TOF MS spectral profiles indicate that 3 strains isolated from the nasal mucosa of healthy adults represent a bacterial species within the genus Bacillus (Figure 2). Striking differences in the peaks of these bacteria were demonstrated. All mass lists were analyzed and compared over the mass interval from 2000 to 20000 Da because of good reproducibility. All final strain-specific peak mass lists were then compared to each other with the application of flexAnalysis (version 3.3) to determine characteristic peak masses.

It should be noted that the peak with the masses at $\mathrm{m} / \mathrm{z}$ $3864 \pm 2,7727 \pm 2$, and $14301 \pm 4$ were exclusively present in the studied strains of Bacillus spp., suggesting that these peaks could represent a genus-specific biomarker. Furthermore, a number of peaks were found in common among the spectral profiles of B. pumilus and B. safensis (4914 $\pm 3,6621 \pm 3$ and $14291 \pm 2$ ), which has no counterpart 


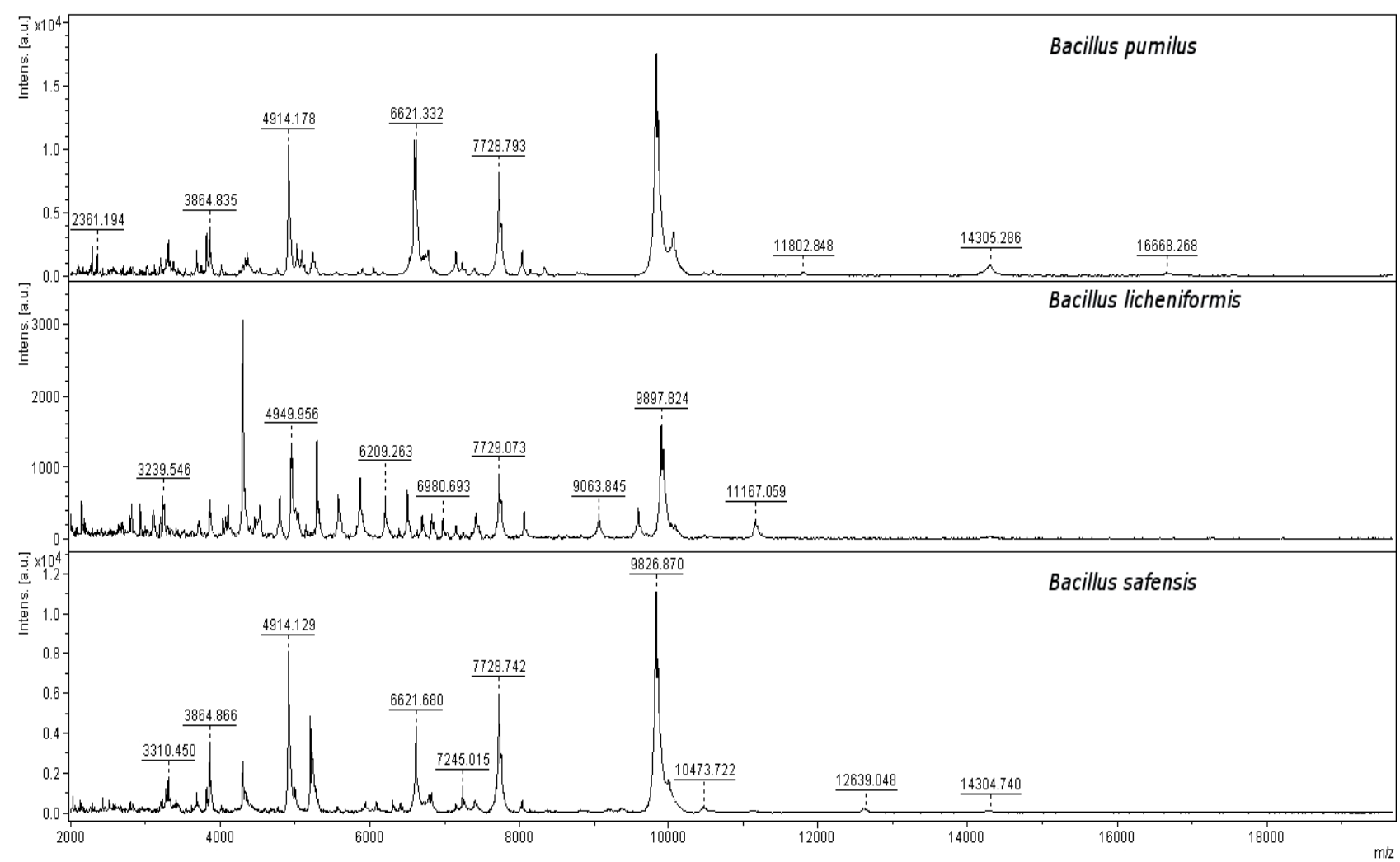

Figure 2. MALDI-TOF mass spectral profiles of B. pumilus, B. licheniformis, and B. safensis tested isolates

in the spectrum of $B$. licheniformis. The presence and/or absence of some peaks and differences in the peak masses indicated intra-genus and intra-species variability, allowing the discrimination between the different species. A number of different peaks were found in the studied Bacillus spp. (B. pumilus - 96, B. licheniformis - 86, B. safensis - 37). For $B$. safensis, other potential biomarkers can be found at $\mathrm{m} / \mathrm{z} 12620$ and for B. pumilus at $\mathrm{m} / \mathrm{z} 16668$.

\section{DISCUSSION}

The upper respiratory tract mucosa membranes provide many different habitats and are colonized by a wide range of microorganisms. The environment in which people live may influence the composition of microbiota. The environment and the animals, e.g. domestic or wild birds, cats, dogs or other organisms, may pose a risk factor as well as be a source of different pathogens or opportunistic bacteria. They can reside in their nasal or throat cavities for a long time without ever causing any clinical signs of disease. For healthy people the risk of catching opportunistic bacteria from domestic birds or other animals and then developing a disease is rare but possible.

Three different Bacillus spp. strains (B. pumilus, B. licheniformis, B. safenis) were identified during our studies with MALDI-TOF MS as microflora colonizing the nasal mucosa of healthy adults living in the suburban area. $\log$ (score) values of the isolated $B$. pumilus, B. safensis and $B$. licheniformis reached 1.78, 2.093 and 2.101, respectively. It means that $B$. pumilus represents the correct identification at the genus level. In contrast to other isolates, a $\log$ (score) value represents a good identification at the species level $(\geq 2)$. Sometimes unique mass profiles are yielded and the results of these bacteria do not match in the database [1].

Bacillus species, e.g. B. marinus, B. subtilis, B. pumilus, $B$. licheniformis, B. cereus, and B. mycoides, are saprophytic aerobic endospore formers widely distributed in the natural environment [9]. These Gram-positive or Gram-variable rods are usually found in soils, vegetable, water, dust, air and deep subterranean sources [19]. B. licheniformis, B. pumilus and $B$. safensis have recently been isolated from marine or other environments, in addition to other species such as B. subtilis or B. cereus [14,23,26,30].

Bacillus spp. may contaminate the hospital environment (hospital linen and dialysis equipment), ventilator equipment and disinfectants $[3,7,11,17]$. Some of these species have been troublesome to food producers and they were also isolated from foodborne infections. Except for a few species, a large majority have no pathogenic potential and have rarely been associated with opportunistic diseases in man or animals.

Some Bacillus spp. are part of the human microbiota. "Nonpathogenic" Bacillus spp. are rarely implicated with human infections but they are among the most widespread microorganisms in the environment and are more frequently isolated as contaminants (in soil, water, air or food) $[14,23,26]$. They have been troublesome to immunocompromised people on account of their environmental presence and resistant endospores or toxins production $[10,25,27,32,33]$. They have been increasingly recognized as opportunistic pathogens, especially in hospitalized patients. Bacillus spp. implicated in opportunistic infections include B. cereus, B. subtilis, B. sphaericus, B. alvei, B. laterosporus, B. licheniformis, B. megaterium and B. pumilus [2-3,5,7,10-11,14,18,21]. 
Identification of bacteria in a clinical microbiology laboratory by using conventional methods are still the gold standard in many laboratories but it is often difficult and takes a long time. Thus, reliable but less complex and modern diagnostic methods of microorganisms isolated from various sources are sought $[6,15,20,22,35]$. Nevertheless, a new identification method using Bruker MALDI-TOF MS system with prior formic acid extraction provided the best diagnostic results and reduced the identification time (often lower than 1 minute) $[4,8,13,15,20,24,28-29,31]$. This kind of method was used previously for the identification and classification e.g. B. pumilus or B. safenis spores [12,16,30]. MALDI-TOF MS technique makes high diagnostic accuracy for a simple, rapid technique for the identification of various types of Gram-positive or Gram-negative bacteria or other organisms. Compared to conventional biochemical and molecular techniques for microorganism identification, MALDI-TOF MS requires minimal sample preparation and achieves more than $92 \%$ correct species identification [34]. Besides, these methods are still expensive and accordingly not available for use under the routine laboratory $[4,13,15,29]$.

\section{CONCLUSIONS}

Using MALDI-TOF MS, a rapid and modern diagnostic technique, the identification of Bacillus spp. and other bacterial isolates (staphylococci and $E$. coli) from the nasal mucosa of healthy adults living in the suburban areas near Lublin was easy to perform. The results of MALDI-TOF MS spectral profiles enabled us to identify B. pumilus, $B$. safensis and $B$. licheniformis, which are difficult for routine diagnostics.

\section{ACKNOWLEDGMENTS}

We thank the director of the Department of Epizootiology and Clinic of Infectious Diseases, Faculty of Veterinary Medicine, University of Life Sciences in Lublin, Stanisław Winiarczyk, for his support. Tomasz Banach is highly acknowledged for excellent technical assistance.

\section{REFERENCES}

1. Alispahic M. et al.: Species-specific identification and differentiation of Arcobacter, Helicobacter and Campylobacter by full-spectral matrix-associated laser desorption/ionization time of flight mass spectrometry analysis. J. Med. Microbiol., 59, 295, 2010.

2. Ball S.C., Sepkowitz K.: Infection due to Bacillus cereus in an injection drug user with AIDS: bacteremia without morbidity. Clin. Infect. Dis., 19, 216, 1994.

3. Barrie D. et al.: Contamination of hospital linen by Bacillus cereus. Epidemiol. Infect., 113, 297, 1994.

4. Bernardo K. et al.: Identification and discrimination of Staphylococcus aureus strains using matrix-assisted laser desorption/ ionization-time of flight mass spectrometry. Proteomics, 2, 747, 2002.

5. Blue S.R., Singh V.R., Saubolle M.A.: Bacillus licheniformis bacteremia: five cases associated with indwelling central venous catheters. Clin. Infect. Dis., 20, 629, 1995.

6. Böhme K. et al.: Species differentiation of seafood spoilage and pathogenic Gram-negative bacteria by MALDI-TOF mass fingerprinting. J. Proteome Res., 9, 3169, 2010.

7. Bryce E.A. et al.: Dissemination of Bacillus cereus in an intensive care unit. Infect. Control Hosp. Epidemiol., 14, 459, 1993.
8. Clark A.E. et al.: Matrix-assisted laser desorption ionization-time of flight mass spectrometry: a fundamental shift in the routine practice of clinical microbiology. Clin. Microbiol. Rev., 26, 547, 2013.

9. Connor N. et al.: Ecology of speciation in the genus Bacillus. Appl. Environ. Microbiol., 76, 1349, 2010.

10. Cotton D.J. et al.: Clinical features and therapeutic interventions in 17 cases of Bacillus bacteremia in an immunosuppressed patient population. J. Clin. Microbiol. 25, 672, 1987.

11. Curtis J.R, Wing A.J., Coleman J.C.: Bacillus cereus bacteraemia - a complication of intermittent haemodialysis. Lancet, 1, 136, 1967.

12. Dickinson D.N. et al.: MALDI-TOF MS compared with other polyphasic taxonomy approaches for the identification and classification of Bacillus pumilus spores. J. Microbiol. Methods, 58, $1,2004$.

13. Dupont C. et al.: Identification of clinical coagulase-negative staphylococci, isolated in microbiology laboratories, by matrixassisted laser desorption/ionization-time of flight mass spectrometry and two automated systems. Clin. Microbiol. Infect., 16, 998, 2010.

14. Farrar W.E.: Serious infections due to "non-pathogenic" organisms of the genus Bacillus. Am. J. Med., 34, 134, 1963.

15. Freiwald A., Sauer S.: Phylogenetic classification and identification of bacteria by mass spectrometry. Nat. Protoc., 4, 732, 2009.

16. Hotta Y. et al.: Classification of the genus Bacillus based on MALDITOF MS analysis of ribosomal proteins coded in S10 and spc operons. J. Agric. Food Chem., 59, 5222, 2011.

17. Hsueh P.R. et al.: Nosocomial pseudo epidemic caused by Bacillus cereus traced to contaminated ethyl alcohol from a liquor factory. J. Clin. Microbiol., 37, 2280, 1999.

18. Ihde D.C., Armstrong D.: Clinical spectrum of infection due to Bacillus species. Am. J. Med., 55, 839, 1973.

19. Ivanova E.P., Mikhailov V.V., Andreev L.A.: Marine bacilli and some approaches to their identification. Mikrobiol. Zhurnal, 54, 27, 1922 (in Russian).

20. Knoester M. et al.: Routine identification of clinical isolates of anaerobic bacteria: matrix-assisted laser desorption ionizationtime of flight mass spectrometry performs better than conventional identification methods. J. Clin. Microbiol., 50, 1504, 2012.

21. Krishnamurthy T., Ross P.L., Rajamani U.: Detection of pathogenic and non-pathogenic bacteria by matrix-assisted laser desorption/ ionization time-of-flight mass spectrometry. Rapid Commun. Mass Spectrom., 10, 883, 1996.

22. Lavigne J.P. et al.: Mass spectrometry: a revolution in clinical microbiology? Clin. Chem. Lab. Med., 36, 1, 2012.

23. Lund B.M.: Foodborne, disease due to Bacillus and Clostridium species. Lancet, 336, 982, 1990.

24. Lynn E.C. et al.: Identification of Enterobacteriaceae bacteria by direct matrix-assisted laser desorptiom/ionization mass spectrometric analysis of whole cells. Rapid Commun. Mass Spectrom., 13, 2022, 1999.

25. O'Day D.M. et al.: The problem of Bacillus infection with special emphasis on the virulence of Bacillus cereus. Ophthalmol., 88, 833, 1981.

26. Oggioni M.R. et al.: Recurrent septicemia in an immunocompromised patient due to probiotic strains of Bacillus subtilis. J. Clin. Microbiol., 36, 325, 1998.

27. Ozkocaman V. et al. Bacillus spp. among hospitalized patients with haematological malignancies: clinical features, epidemics and outcomes. J. Hosp. Infect., 64, 169, 2006.

28. Pribil P., Fenselau C.: Characterization of enterobacteria using MALDI-TOF mass spectrometry. Anal. Chem., 77, 6092, 2005.

29. Rajakaruna L. et al.: High throughput identification of clinical isolates of Staphylococcus aureus using MALDI-TOF MS of intact cells. Infect. Genet. Evol., 9, 507, 2009.

30. Satomi M., La Duc M.T., Venkateswaran K.: Bacillus safensis sp. nov., isolated from spacecraft and assembly-facility surfaces. Int. J. Syst. Evol. Microbiol., 56, 1735, 2006.

31. Sauer S. et al.: Classification and identification of bacteria by mass spectrometry and computational analysis. PLoS ONE, 3, e2843, 2008. (doi: 10.1371/journal.pone. 0002843)

32. Sorokulova I.: Modern status and perspectives of Bacillus bacteria as probiotics. J. Prob. Health., 1, 1000e106, 2013. (doi: 10.4172/2329-8901.1000e106) 
33. Tuazon C.U. et al.: Serious infections from Bacillus sp. JAMA, 241, 1137, 1979.

34. Van Veen S., Claas E., Kuijper E.J.: High-throughput identification of bacteria and yeast by matrix-assisted laser desorption ionizationtime of flight mass spectrometry in conventional medical microbiology laboratories. J. Clin. Microbiol., 48, 900, 2010.
35. Wolters M. et al.: MALDI-TOF MS fingerprinting allows for discrimination of major methicillin-resistant Staphylococcus aureus lineages. Int. J. Med. Microbiol., 301, 64, 2011. 